\title{
Gpx5 protects the family jewels
}

\author{
R. John Aitken
}

ARC Centre of Excellence in Biotechnology and Development, Discipline of Biological Sciences, University of Newcastle, Newcastle, New South Wales, Australia.

\begin{abstract}
Immature spermatozoa are vulnerable to oxidative stress after their release from the testes, due in part to an innate deficiency in antioxidant enzymes. The male reproductive tract compensates for this deficiency by secreting antioxidant enzymes such as glutathione peroxidase $5(\mathrm{Gpx} 5)$ into the epididymal lumen. In this issue of the JCI, Chabory et al. examined the phenotype of $\mathrm{Gpx}^{-/-}$mice and found that while deletion of this gene did not seem to affect fertility per se, it did influence the incidence of miscarriage and embryonic defects in mated wild-type female mice (see the related article beginning on page 2074). Importantly, the appearance of these problems was age dependent and associated with signs of oxidative stress in the spermatozoa. These results demonstrate the key importance of $\mathrm{Gpx} 5$ as an extracellular antioxidant designed to protect maturing mammalian spermatozoa from oxidative stress.
\end{abstract}

Imagine you are a spermatozoon. As an immature germ cell, you lived your life within the seminiferous tubules of the testes, in cytoplasmic communion with your brothers and sisters, nourished and nurtured by Sertoli cells (Figure 1). Then, just when you had completed the complexities of meiosis and your gene transcription machinery had closed down, you were forced to undergo one of the most dramatic morphological transformations in biology - the metamorphosis of a small, spherical haploid cell, the round spermatid, into the epitome of specialized cell types, the spermatozoon. Once created, you were then cast free from the germinal epithelium in a carefully regulated process known as spermiation. At this point in your life history you have, apart from a little too much cytoplasm around your waist, the outward appearance of a mature, functional spermatozoon. However, in reality, you are neither of these things. You may look like a spermatozoon, but you have none of the functional attributes of this cell; you cannot swim, cannot recognize the egg, and cannot engage in the complex array of cell-cell interactions that lead to fertilization. You have virtually no cytoplasm, no capacity for DNA repair, and little facility for apoptosis. And from now on, you are on your own.

Conflict of interest: The author has declared that no conflict of interest exists.

Nonstandard abbreviations used: Gpx5, glutathione peroxidase 5 .

Citation for this article: $J$. Clin. Invest. 119:1849-1851 (2009). doi:10.1172/JCI39688.
Spermatozoon functionality is acquired during two major phases of post-testicular maturation. The first phase involves passage through a complex, convoluted, regionally differentiated organ known as the epididymis (Figure 1). Within this organ, spermatozoa acquire the potential for progressive movement and fertilization. However, as long as they remain within the epididymal lumen, this potential is never realized. Instead, these cells are stored in a potentially functional but quiescent state, exhibiting little, if any, movement, until the moment of ejaculation. At this point spermatozoa suddenly explode into a state of vigorous progressive motility that allows these cells to begin their ascent of the female reproductive tract to the site of fertilization, high in the fallopian tubes. During this journey spermatozoa undergo the second phase of their maturation known as capacitation, which allows these cells to express the range of potential functions acquired during epididymal maturation. These functional attributes are illustrated in Figure 1 and include: (a) a specialized form of movement known as byperactivation that facilitates the final stages of sperm transport and penetration of the zona pellucida, a thick shell surrounding the oocyte; (b) an exquisitely specific cell-cell recognition event that allows the spermatozoon to recognize a single cell, the oocyte, while ignoring the hundreds of thousands of other cells encountered on their journey through the female tract; (c) a secretory event, known as the acrosome reaction that releases enzymes to further facilitate zona penetration; (d) penetration of the zona matrix; and (e) remodeling of a specific region of the plasma membrane in the equatorial segment of the sperm head so that it will recognize, and fuse with, the surface of the oocyte (Figure 1).

Remarkably, the highly complex series of changes depicted in Figure 1 occur in a cell that is transcriptionally and translationally silent and driven entirely by posttranslational protein modifications. Furthermore, this maturation process involves a week or more of isolated existence for the spermatozoa as they transit the epididymis, followed by a further period of isolation in the female reproductive tract as the cells capacitate (a process by which the spermatozoon becomes capable of fertilizing an egg) and wait for an egg to arrive.

\section{Spermatozoa are vulnerable to oxidative stress}

Throughout the whole of this period, spermatozoa are vulnerable to attack from ROS. This vulnerability is partly due to the fact that these cells are loaded with substrates for free radical attack, including polyunsaturated fatty acids and DNA $(1,2)$. These cells are also professional generators of ROS. Indeed, they were the first cell type ever shown to generate ROS, as described in a Nature paper published in 1946 (3), predating the discovery of this activity in leukocytes by more than a decade. In addition, spermatozoa adopt the unusual strategy of divesting themselves of most of their cytoplasm during spermiogenesis, thereby shedding a key cellular constituent that, in all other cell types, offers protection against oxidative stress through the presence of specialized antioxidant enzymes such as catalase, superoxide dismutase, and glutathione peroxidase (Gpx).

While the highly compacted nature of mammalian spermatozoa confers upon these cells some resistance against oxidative damage (4), they are exquisitely dependent on the antioxidant properties of the surrounding fluids to protect them from ROS attack. It is for this reason that both epididymal and seminal plasma 


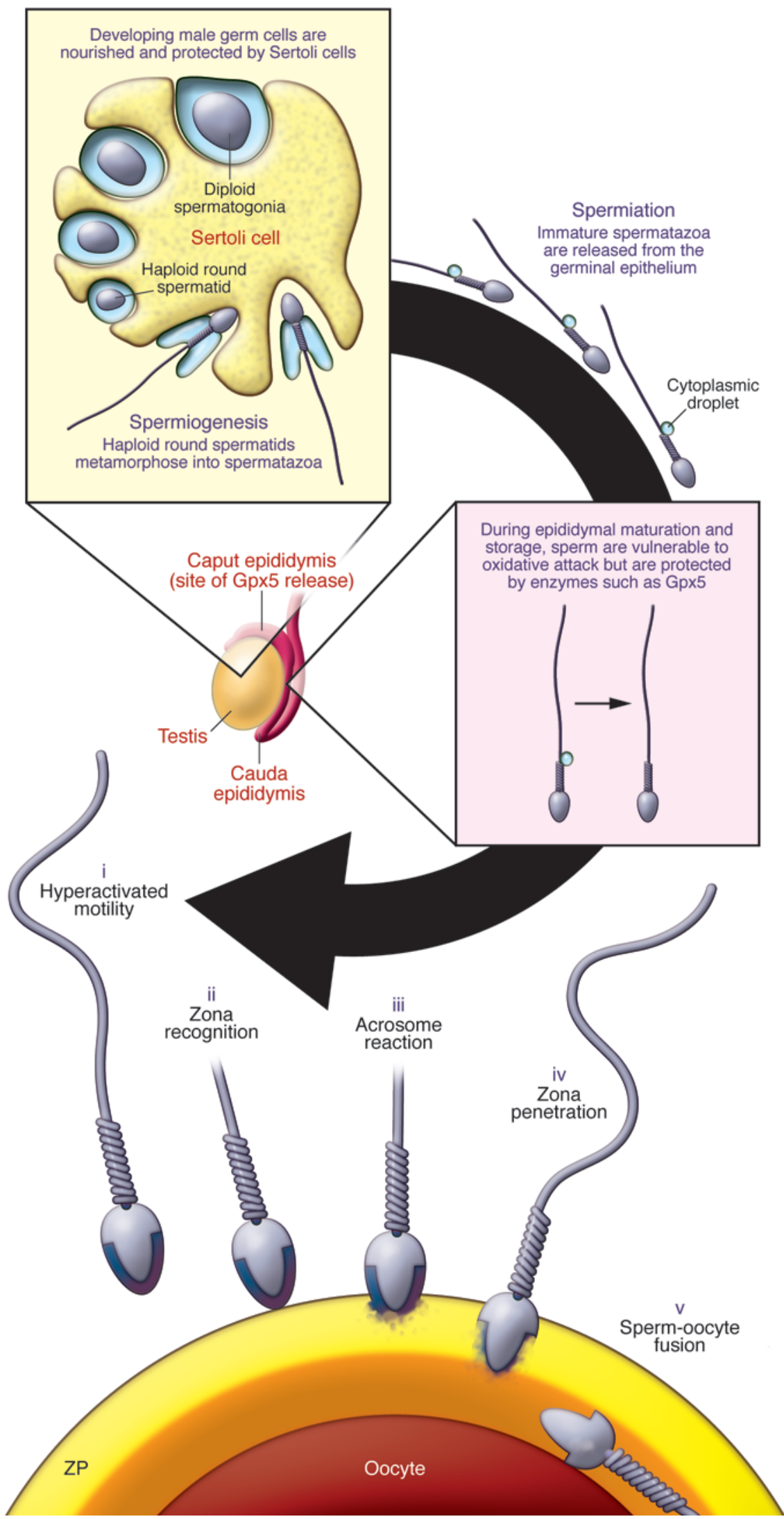

\section{Figure 1}

A simplified life history of mammalian spermatozoa. Male germ cells differentiate in the germinal epithelium, nourished and protected by the nurse cells of the testes, Sertoli cells. Spermatozoa emerge from meiosis as a syncytium of small round spermatids that, in the absence of de novo gene transcription, undergo one of the most dramatic transformations in biology, as they engage in a process known as spermiogenesis and transform into spermatozoa. Once spermiogenesis has been completed, spermatozoa leave the germinal epithelium structurally complete (apart from a cytoplasmic droplet in the sperm midpiece) but lacking in functional competence. The potential to express movement and fertilize the oocyte is acquired during epididymal transit. Spermatozoa are then stored in a quiescent state in the cauda epididymis, ready for ejaculation and subsequent capacitation in the female tract. As a result of capacitation, spermatozoa realize the range of functions acquired as a result of epididymal maturation, including (i) hyperactivated motility, (ii) zona recognition, (iii) the acrosome reaction, (iv) zona penetration, and (v) sperm-oocyte fusion. Throughout their maturation in the epididymis, the spermatozoa exist as isolated cells that are vulnerable to oxidative attack. Because they lack substantial intrinsic defense mechanisms, these cells are highly dependent on extracellular antioxidants released into the epididymal lumen. Prominent among the complex array of antioxidant enzymes present in epididymal plas$\mathrm{ma}$ is $\mathrm{Gpx} 5$. In this issue of the $\mathrm{JCl}$, Chabory et al. (7) report that deletion of Gpx5 in male mice generates a state of oxidative stress within the epididymis that becomes particularly marked with age and is associated with both miscarriage and developmental defects in the embryos. ZP, zona pellucida. 
are highly enriched with antioxidants, including small-molecular-mass free radical scavengers (vitamin C, uric acid, taurine, thioredoxin) and highly specialized extracellular antioxidant enzymes, including unique isoforms of superoxide dismutase and Gpx, particularly Gpx5 (5).

Gpx5 is an unusual Gpx, in that it is solely expressed in the caput epididymis under androgenic control. It is also unusual in that it lacks a selenocysteine residue, while still retaining its antioxidant properties (6). This protein associates with the sperm surface during epididymal transit and protects the spermatozoa from peroxide-mediated attack while they are undergoing maturation $(5,6)$. The functional significance of this enzyme has been beautifully illustrated by a landmark paper in this issue of the JCI by Chabory et al. describing the phenotype of the Gpx5-knockout mouse (7). Although deletion of the Gpx5 gene did not appear to have any effect on male fertility, it did result in a high rate of miscarriage in mated wildtype female mice, as well as developmental defects in the offspring. Furthermore, these changes were only observed in embryos sired by Gpx5-deficient mice more than one year old and were associated with increased oxidative damage in the spermatozoa. The onset of this pathology was delayed because of a concomitant upregulation of alternative antioxidant genes in the epididymis, including other isoforms of Gpx and catalase (Cat), in response to the increased oxidative stress.

\section{Clinical relevance}

These findings have substantial clinical relevance. The levels of sperm DNA damage are often markedly elevated in cases of male infertility $(2,8)$. This damage is also associated with a wide range of adverse clinical outcomes, including impaired fertilization, disrupted preimplantation embryonic development, and, as was observed in the current study of male Gp $x 5^{-/-}$mice (7), increased rates of miscarriage and offspring morbidity $(1,2,8,9)$. Although these adverse clinical effects are not always observed with the same degree of penetration in independent clinical studies, the overall weight of evidence suggests that oxidative DNA damage in the male germ line is potentially damaging for both the progress of pregnancy and the health and wellbeing of the offspring. Moreover, like the Gpx $55^{-1-}$ mice, the DNA damage we see in human spermatozoa is commonly oxidative in nature (10) and is positively associated with male age $(11,12)$. Furthermore, recent studies in an animal model for aging studies, the brown Norway rat, have confirmed that increased paternal age is associated with increased levels of DNA damage and oxidative stress in epididymal spermatozoa (13).

The clinical consequences of this DNA damage to sperm can be seen in the wide variety of pathologies that have been observed in children and young adults in association with advanced paternal age, including dominant genetic diseases and complex polygenic neurological conditions such as bipolar disease, epilepsy, spontaneous schizophrenia, and autism $(2,8,14-16)$. In keeping with these data, the experimental injection of DNA-damaged spermatozoa into mouse oocytes has not only been shown to affect embryo development but also to significantly affect the behavior, postnatal growth, and longevity of the offspring as well as their susceptibility to tumors (17). These data also echo previous reports associating paternal smoking, oxidative damage to sperm DNA, and the incidence of cancer in children $(18,19)$.

Under physiological circumstances, spermatozoa possessing oxidatively damaged DNA would be prevented from fertilizing the oocyte because collateral peroxidative damage to the sperm plasma membrane would disrupt the process of sperm-oocyte fusion (20). However, when intracytoplasmic sperm injection (ICSI) is used as the in vitro fertilization technique, such biological barriers to fertilization are completely bypassed (21). Since the use of such assisted reproductive technologies is rising exponentially, it is highly likely that conceptions involving oxidatively damaged spermatozoa are a relatively common occurrence in current clinical practice. The data generated by Chabory et al. (7) serve to remind us that the health and well-being of children generated in assisted conception clinics is at risk and should be carefully monitored, or we will have to deal with the consequences in the years to come.

Address correspondence to: R. John Aitken, ARC Centre of Excellence in Biotechnology and Development, Discipline of Biological Sciences, Room LS4.32, University of Newcastle, Callaghan, New South Wales, Australia. Phone: 61-2-4921-2082; Fax: 61-2-4921-6308; E-mail: john.aitken@ newcastle.edu.au.
1. Aitken, R.J. 1999. The Amoroso Lecture. The human spermatozoon - a cell in crisis? J. Reprod. Fertil. 115:1-7.

2. Aitken, R.J., De Iuliis, G.N., and McLachlan, R.I. 2009. Biological and clinical significance of DNA damage in the male germ line. Int. J. Androl. 32:46-56.

3. Tosic, J., and Walton, A. 1946. Formation of hydrogen peroxide by spermatozoa and its inhibitory effect on respiration. Nature. 158:485.

4. Sawyer, D.E., Mercer, B.G., Wiklendt, A.M., and Aitken, R.J. 2003. Quantitative analysis of gene-specific DNA damage in human spermatozoa. Mutat. Res. 529:21-34.

5. Drevet, J.R. 2006. The antioxidant glutathione peroxidase family and spermatozoa: a complex story. Mol. Cell. Endocrinol. 250:70-79.

6. Vernet, P., Rigaudiére, N., Ghyselinck, N., Dufaure, J.P., and Drevet, J.R. 1996. In vitro expression of a mouse tissue specific glutathione-peroxidase-like protein lacking the selenocysteine can protect stably transfected mammalian cells against oxidative damage. Biochem. Cell. Biol. 74:125-131.

7. Chabory, E., et al. 2009. Epididymis selenoindependent glutathione peroxidase 5 maintains sperm DNA integrity in mice. J. Clin. Invest. 119:2074-2085.

8. Aitken, R.J., and De Iuliis, G.N. 2007. Origins and consequences of DNA damage in male germ cells. Reprod. Biomed. Online. 14:727-733.

9. Evenson, D., and Wixon, R. 2006. Meta-analysis of sperm DNA fragmentation using the sperm chromatin structure assay. Reprod. Biomed. Online. 12:466-472.

10. Kodama, H., Yamaguchi, R., Fukuda, J., Kasi, H., and Tanak, T. 1997. Increased deoxyribonucleic acid damage in the spermatozoa of infertile male patients. Fertil. Steril. 68:519-524.

11. Schmid, T.E., et al. 2007. The effects of male age on sperm DNA damage in healthy non-smokers. Hum. Reprod. 22:180-187.

12. Singh, N.P., Muller, C.H., and Berger, R.E. 2003. Effects of age on DNA double-strand breaks and apoptosis in human sperm. Fertil. Steril. 80:1420-1430.

13. Weir, C.P., and Robaire, B. 2007. Spermatozoa have decreased antioxidant enzymatic capacity and increased reactive oxygen species production during aging in the Brown Norway rat. J. Androl. 28:229-240.

14. Sipos, A., et al. 2004. Paternal age and schizophrenia: a population based cohort study. BMJ. 329:1070.

15. Reichenberg, A., et al. 2006. Advancing paternal age and autism. Arch. Gen. Psychiatry. 63:1026-1032.

16. Frans, E.M., et al. 2008. Advancing paternal age and bipolar disorder. Arch. Gen. Psychiatry. 65:1034-1040.

17. Fernández-Gonzalez, R., et al. 2008. Long-term effects of mouse intracytoplasmic sperm injection with DNA-fragmented sperm on health and behavior of adult offspring. Biol. Reprod. 78:761-772.

18. Fraga, C.G., Motchnik, P.A., Wyrobek, A.J., Rempel, D.M., and Ames, B.N. 1996. Smoking and low antioxidant levels increase oxidative damage to DNA. Mutat. Res. 351:199-203.

19. Ji, B.T., et al. 1997. Paternal cigarette smoking and the risk of childhood cancer among offspring of nonsmoking mothers. J. Natl. Cancer Inst. 89:238-244.

20. Aitken, R.J., Clarkson, J.S., and Fishel, S. 1989 Generation of reactive oxygen species, lipid peroxidation, and human sperm function. Biol. Reprod. 41:183-197.

21. Twigg, J.P., Irvine, D.S., and Aitken, R.J. 1998. Oxidative damage to DNA in human spermatozoa does not preclude pronucleus formation at intracytoplasmic sperm injection. Hum. Reprod. 13:1864-1871. 